\title{
Accident Positioning System
}

\author{
Shiv Prakash Gupta \\ B.E \\ EPCET Bangalore \\ India
}

\author{
Vishwa Mohan Prasad \\ M.Tech Student \\ NRIT Hderabad \\ India
}

\author{
Anita R. \\ Assoc. Professor, \\ EPCET Bangalore \\ India
}

\begin{abstract}
Recently technological and population development, the usage of vehicles are rapidly increasing and at the same time the rate of accidents are also increasing. Hence, the value of human life is ignored. No one can prevent the accident, but can save their life by expediting the ambulance to the hospital in time. A new vivid scheme called Accident Positioning System (APS) is introduced. The objective of this scheme is to minimize the delay in providing emergency medical services after accidents. The concept of this scheme is to send the location information to the ambulance, hospital, friends, relatives immediately after the accident. So that the ambulance can reach the spot in time and human life can be saved. The android application finds the location using the received message and sends the exact accident location to the registered database (emergency contact numbers like friends, relatives, ambulance). This scheme is fully automated, thus it locates the accident spot accurately and immediately notifies us Your general terms must be any term which can be used for general classification of the submitted material such as Pattern Recognition, Security, Algorithms et. al.
\end{abstract}

\section{General Terms GSM. GPS.}

\section{Keywords}

Counter, ROM, CMOS, ALU,CPU, Max232, Memory

\section{INTRODUCTION}

There is a drastic increase in the number of vehicles in these days which also cause a steep rise in the number of accidents with a lot of people losing their lives. According to the World Health Organization, an estimated 1.2 million people lose their lives every year due to car accidents. India's road accident records $16 \%$ of the world's road accident deaths, whereas India has only $1 \%$ of the world's road vehicles. It is due to the increase in the number of vehicles without a subsequent increase in the road facilities required for it. In most of the accident cases, the victims lose their lives because of the unavailability of medical facilities at the right time. To solve problems like these, this project came into existence.

\section{RELECTED WORK}

\subsection{Working}

This project is mainly used to track the position of the Vehicle by the friends,relatives, ambulances to provide emergency medical services. In case of any accident, the system sends automated messages to the pre-programmed numbers. We can send messages to any number of mobiles. The location details is sent from the vehicle to, Police to clear the traffic, Ambulance to save the people using message received by this device. This uses a GPS (Global Positioning System) to know the exact position of the vehicle with an accuracy of a few feet. GSM is used to send an SMS from the device to get position of the vehicle through the GPS.This paragraph is a repeat of 3.1 Industries not traditionally known to use vehicle tracking systems have started to use it in creative ways to improve their processes or businesses. The hospitality industry has caught on to this technology to improve customer service. For example, a luxury hotel in Singapore has been known to install vehicle tracking system in their limousines to ensure they can welcome their VIPs when they reach the hotel. Vehicle tracking systems have also been used in food delivery and car rental companies.

\subsection{The Working Of The Device Is Done In Two PartsSender Module (installed in the vehicle):}

Whenever an accident occurs there is a deformation in the metal surface of the vehicle. The inductive proximity sensor (metal sensor) senses any deformation in the metal surface of the vehicle and informs the microcontroller that an accident has occurred. The microcontroller activates the GPS which records the current latitude and longitude information and sends it to the microcontroller. The microcontroller has the registered database of the mobile numbers where a text message will be sent. The message can be sent to any number of mobile numbers. This database contains the mobile numbers of relatives,friends, ambulance and hospital. The microcontroller activates the GSM module and instructs it to send this latitude longitude information to the registered mobile numbers. The microcontroller 8051 controls and coordinates all the processes of the system. The receiver system (Android Mobile) The registered numbers gets the latitude and longitude information where an android application checks for errors and finds the exact location send and shows it on the google map that where the accident has occurred. Microcontroller is the major component of the system also called as a computer on chip which has features of a Microprocessor like CPU, ALU, PC, SP and Registers. It also has added the other features needed to make complete computer like ROM, RAM, Serial and Parallel I/O, counters and Clock circuit.

Like the Microprocessor, a Microcontroller is a generalpurpose device, but one that is mean to read data, performs limited calculation on that data, and control its environment based on those calculations. The prime use of Microcontroller is to control the operation of a machine using a fixed program that is stored on ROM and that does not change over the life time of the system. The AT89S52 is a low-power, highperformance CMOS 8-bit microcontroller with $8 \mathrm{~K}$ bytes of insystem programmable Flash memory. The device is manufactured using Atmel's high-density nonvolatile memory technology and is compatible with the industry- standard $80 \mathrm{C} 51$ instruction set and pinout.

This project is custom made for the heavily populated countries like India. It is used to save the person's life by making the medical facilities arriving in time. The location of the vehicle can be known by using a GPS receiver. After accident happens the response is sent to friends, relatives and 
hospital, which is already registered in the database via a GSM modem. An inductive proximity sensor is used to detect any accident which will trigger a signal in case of any accidents. The microcontroller controls and coordinates all the operations in the system. MAX232 is used for the communication between the different components in the system. A latch for separating address from multiplex address and data.32-KB RAM and 32-KB ROM to be able to satisfy most applications. And also Timer / Counter, Parallel programmable port, Serial port, and Interrupt controller are needed for its efficient applications.

In comparison a typical Micro controller 8051 chip has all that the 8051 board has except a reduced memory as follows. Slower Development time: As we have observed Microprocessors need a lot of debugging at board level and at program level, whereas, Micro controller do not have the excessive circuitry and the built-in peripheral chips are easier to program for operation.

So peripheral devices like Timer/Counter, Parallel programmable port, Serial Communication Port, Interrupt controller and so on, which were most often used were integrated with the Microprocessor to present the Micro controller .RAM and ROM also were integrated in the same chip. The ROM size was anything from 256 bytes to $32 \mathrm{~Kb}$ or more. RAM was optimized to minimum of 64 bytes to 256 bytes or more.

Port 0 is an 8-bit open drain bidirectional $\mathrm{I} / \mathrm{O}$ port. As an output port, each pin can sink eight T.TL inputs. When $1 \mathrm{~s}$ are written to port 0 pins, the pins can be used as high-impedance inputs. Port 0 can also be configured to be the multiplexed low-order address/data bus during accesses to external program and data memory. In this mode, $\mathrm{P} 0$ has internal pullups. Port 0 also receives the code bytes during Flash programming and outputs the code bytes during program verification. External pull-ups are required during program verification.

\section{DESIGN}

A Micro controller consists of a powerful CPU tightly coupled with memory, various I/O interfaces such as serial port, parallel port timer or counter, interrupt controller, data acquisition interfaces-Analog to Digital converter, Digital to Analog converter, integrated on to a single silicon chip. If a system is developed with a microprocessor, the designer has to go for external memory such as RAM, ROM, EPROM and peripherals. But controller is provided all these facilities on a single chip. Development of a Micro controller reduces PCB size and cost of design. One of the major differences between a Microprocessor and a Micro controller is that a controller often deals with bits not bytes as in the real world application. Intel has introduced a family of Micro controllers called the MCS-51.The microcontroller plays the major role in any embedded project. In this my project we use two microcontrollers they are made by the ATMEL Company. That is AT89S52. Port 1 is an 8-bit bidirectional I/O port with internal pull-ups. The Port 1 output buffers can sink/source four TTL inputs. When $1 \mathrm{~s}$ are written to Port 1 pins, they are pulled high by the internal pull-ups and can be used as inputs. As inputs, Port 1 pins that are externally being pulled low will source current (IIL) because of the internal pull-ups. In addition, P1.0 and P1.1 can be configured to be the timer/counter 2 external count input (P1.0/T2) and the timer/counter 2 trigger input $(\mathrm{P} 1.1 / \mathrm{T} 2 \mathrm{EX})$, respectively, as shown in the following table
4. T2EX (Timer/Counter 2 capture/reload trigger and direction

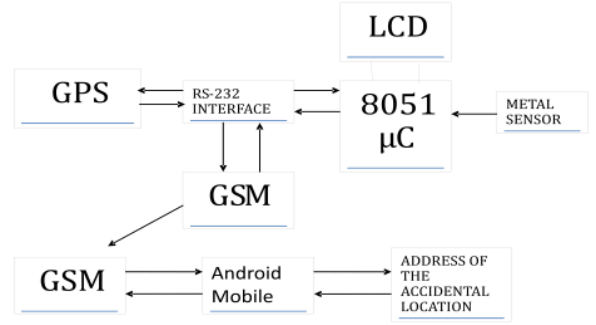

Fig 1: Block Diagram

\section{IMPLEMENTION}

The AT89S52 is designed with static logic for operation down to zero frequency and supports two software selectable power saving modes. The Idle Mode stops the CPU while allowing the RAM; timer/counters, serial port, and interrupt system to continue functioning. The Power-down mode saves the RAM contents but freezes the oscillator disabling all other chip functions until the next hardware reset.

\subsection{Keil Software}

Keil development tools for the 8051 Microcontroller Architecture support every level of software developer from the professional applications engineer to the student just learning about embedded software. The industry-standard Keil C Compilers, Macro Assemblers, Debuggers, Real-time Kernels, Single-board Computers, and Emulators support all 8051 derivatives and help you get your projects completed on schedule. The $\mu$ Vision Simulator allows you to debug programs using only your PC using simulation drivers provided by Keil and various third-party developers. A good simulation environment, like $\mu$ Vision, does much more than simply simulate the instruction set of a microcontroller - it simulates your entire target system including interrupts, startup code, on-chip peripherals, external signals, and I/O. Use of software for execution of microcontroller programs :Keil development tools for the MC architecture support every level of software developer from the professional applications engineer to the student just learning about embedded software development. The industry-standard Keil-C compilers, macro assemblers, debuggers, real, time Kernels, Single-board computers and emulators support all microcontroller derivatives and help you to get more projects completed on schedule. The Keil software development tools are designed to solve the complex Problems facing embedded software developers. Those are listed below. Keil development tools for the MC architecture support every level of software developer from the professional applications engineer to the student just learning about embedded software development. The industry-standard Keil-C compilers, macro assemblers, debuggers, real, time Kernels, Single-board computers and emulators support all microcontroller derivatives and help you to get more projects completed on schedule. The Keil software development tools are designed to solve the complex Problems facing embedded software developers. Those are listed below. 


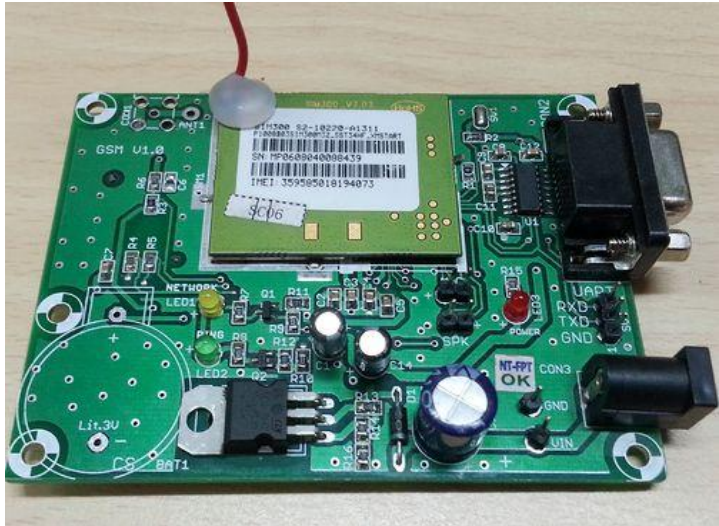

Figure 2: GSM Module

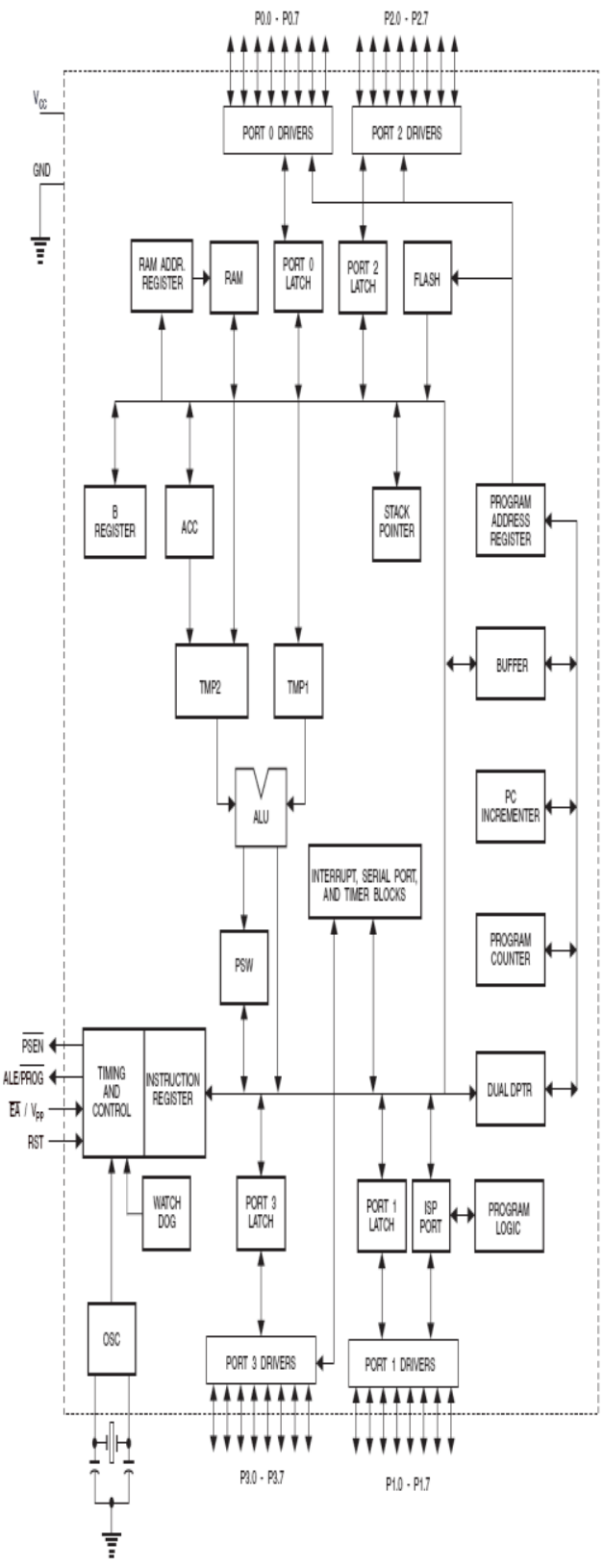

Figure 3: Circuit Of Microcontroller
The 256 byte internal RAM is organized into 3 distinct areas. 32 bytes from $00 \mathrm{~h}$ to $1 \mathrm{Fh}$ that make up 32 working registers are organized as 4 banks of 8 registers each. The 4 register banks are numbered 0 to 3 and are made up of 8 registers R0 to R7. Each register can be addressed by the name of the bank or by its RAM address. Thus R0 of bank-3 is R0 (if bank-3 is currently selected) or address $18 \mathrm{~h}$ (whether bank-3 is selected or not). Bits RS0 and RS1 in the PSW determine which bank of registers is currently in use at any time when the program is running. Register banks not selected can be used as general purpose RAM. Bank-0 is selected on reset. A bit addressable area of 16 bytes occupies RAM byte addresses $20 \mathrm{~h}$ to $2 \mathrm{Fh}$, forming a total of 128 addressable bits. An addressable bit may be specified by its bit address of $00 \mathrm{~h}$ to $7 \mathrm{Fh}$, or 8 bits may form any byte address from $20 \mathrm{~h}$ to $2 \mathrm{Fh}$. Addressable bits are useful when the program need only remember a binary event2.GSM Program

points of white space above the subsubsection head.

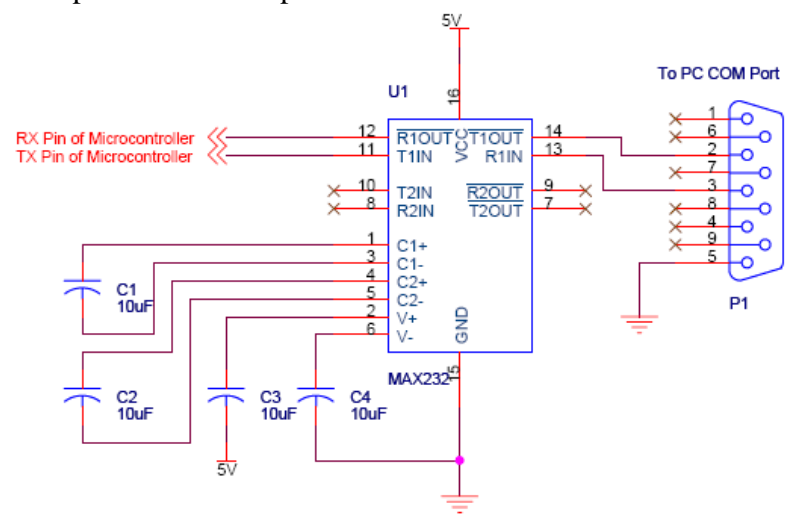

Figure 4: GSM Circuit

The following table lists the AT commands that are related to the writing and sending of SMS messages

\section{Table 1.GSM AT Commands}

$\begin{array}{ll}\text { AT command Meaning } \\ \text { +CMGS } & \text { Send message } \\ \text { +CMSS } & \text { Send message from storage } \\ \text { +CMGW } & \text { Write message to memory } \\ + \text { +CMGD } & \text { Delete message } \\ \text { +CMGC } & \text { Send command } \\ + \text { CMMS } & \text { More messages to send }\end{array}$

\section{PROGRAM LCD Program:} \#include "lcd.h"

\#include<reg51.h>

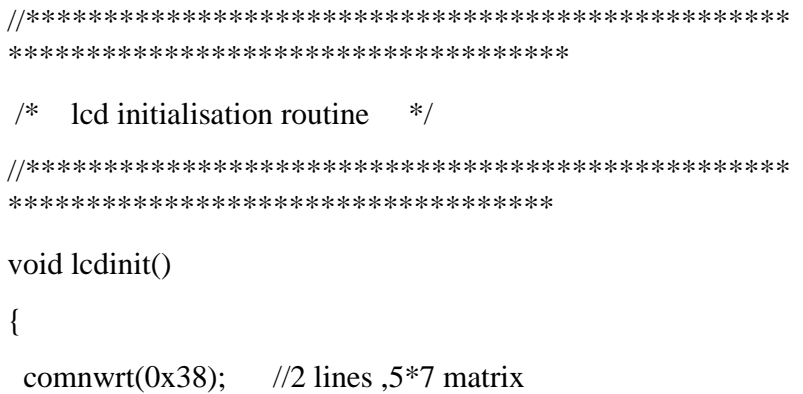

lcd_delay(); 
$/ / \mathrm{a}=0 \mathrm{x} 0 \mathrm{e} ; \quad / / \mathrm{screen}$ and cursor on no blink

comnwrt(0x0e);

lcd_delay();

$/ / \mathrm{a}=0 \mathrm{x} 01 ; \quad$ //clear lcd

comnwrt(0x01);

lcd_delay();

comnwrt(0x06);

lcd_delay();

//comnwrt(0x84);

//lcd_delay();

\}

void comnwrt(unsigned char a)

\{

LCD_DATA_PORT $=$ a;

LCD_RS $=0$;

$0 \quad \mathrm{RS}$

$/ /$ port2 , bit

//LCD_RW=0;

$1 \mathrm{R} / \mathrm{W}$

$\mathrm{LCD} E=1$;

LCD_E $=0$;

$/ / * * * * * * * * * * * * * * * * * * * * * * * * * * * * * * * * * * * * * * * * * * * * * * * * *$

******************************************

/* lcd display routine for a character */

$/ / * * * * * * * * * * * * * * * * * * * * * * * * * * * * * * * * * * * * * * * * * * * * * * * * *$ $* * * * * * * * * * * * * * * * * * * * * * * * * * * * * * * * * * *$

void display1(unsigned char c)

\{

LCD_DATA_PORT=c;

LCD_RS=1; //port2 bit0 RS

//LCD_RW=0; $/ /$ port2 , bit $1 \quad \mathrm{R} / \mathrm{W}$

LCD_E=1; //port2 , bit 2 E

LCD_E $=0$;

lcd_delay();

$$
\text { \} }
$$$$
\text { \} }
$$

$/ / * * * * * * * * * * * * * * * * * * * * * * * * * * * * * * * * * * * * * * * * * * * * * * * * *$ ******************************************

/* lcd display routine for a string */

$/ / * * * * * * * * * * * * * * * * * * * * * * * * * * * * * * * * * * * * * * * * * * * * * * * * *$
$* * * * * * * * * * * * * * * * * * * * * * * * * * * * * * * * * * *$

void display(char *str)

\{

// $\quad$ char counter $=0$

while $(*($ str $) !=' \backslash 0 ')$

\{

/* $\quad$ counter++;

if $($ counter $==15)$

\{

nextline();

\} $\quad * /$

LCD_DATA_PORT=*(str);

LCD_RS=1;

//port2 bit0

RS

//LCD_RW=0; $/ /$ port2 , bit $1 \quad \mathrm{R} / \mathrm{W}$

LCD_E $=1$;

$/ /$ port 2 , bit $2 \quad \mathrm{E}$

LCD_E=0;

$/ /$ port 2 , bit 2

lcd_delay();

str++;

\}

\}

$/ / * * * * * * * * * * * * * * * * * * * * * * * * * * * * * * * * * * * * * * * * * * * * * * * * *$

$* * * * * * * * * * * * * * * * * * * * * * * * * * * * * * * * * * * *$

/* moves the lcd cursor to the next line */

//****************************************************** $* * * * * * * * * * * * * * * * * * * * * * * * * * * * * * * * * * *$

void nextline()

\{

comnwrt(0xc0);

lcd_delay();

\}

| $* * * * * * * * * * * * * * * * * * * * * * * * * * * * * * * * * * * * * * * * * * * * * * * * *$ ***************************************

move to previous line

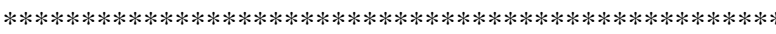
***********************************/

/*void previousline()

\{

comnwrt(0x80);

lcd_delay(); 


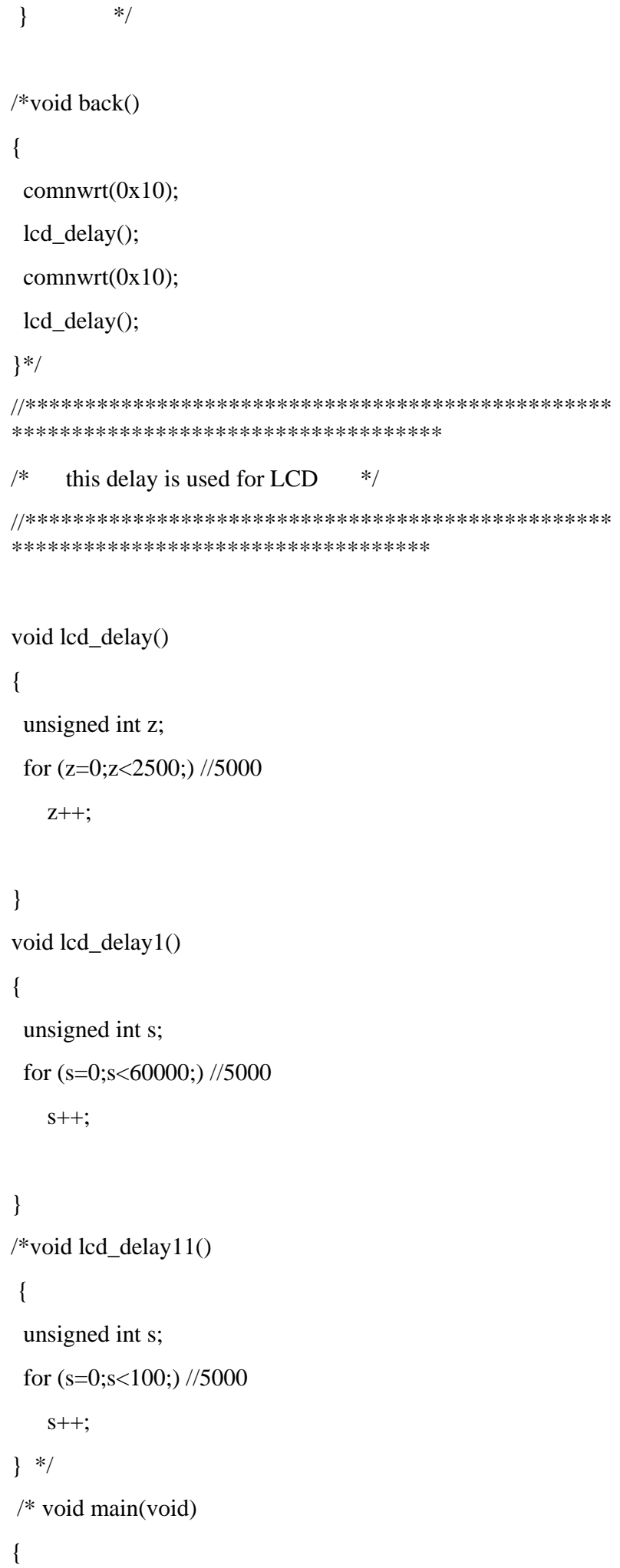

\section{CONCLUSION AND FUTURE WORK}

The main objective of this project is to provide immediate medical services in order to save lives after accidents. To take immediate action when an accident occurs by alerting the respective people by sending message Security System

In future we can use this system can be interfaced with Vehicle Airbag System This can also be developed by interconnecting a camera to the controller module that takes the photograph of the accident spot that makes tracking easier

\section{ACKNOWLEDGMENTS}

We are grateful to our internal guide Mrs. Anita R, Associate Professor, Department of ECE, EPCET for her assistance throughout our project work We are greatly indebted to our external guide Mr. Vikash Kumar, EC Engg., S2P Academy,for his full support and guidance throughout the entire project work. We would also like to thank all people who have extended their help and cooperation

\section{REFERENCES}

[1] Adnan I. Yaqzan, Issam W. Damaj, and Rached N. Zantout (July 24, 2008), ' GPSBased Vehicle Tracking System-On-Chip', Proceedings of the world Congress on Engineering Vol I WCE

[2] Arias Tanti Hapsari Eniman Y Syamsudin Imron Pramana (2005) ,'Design Of Vehicle

[3] Position Tracking System Using Short Message Services And Its Implementation On

[4] FPGA', Proceedings of the 2005 Asia and South Pacific Design Automation Conference, ISBN:0-7803-8737-6

[5] Peter J. Ashenden (1995) ,'The designer's guide to VHDL', Morgan Kaufmann Publishers,San Francisco

[6] Theodore S. Rappaport (2008), 'Wireless Communication', Prentice Hall PTR2 .

[7] Vijay Kumar Garg, Joseph E. Wilkes (October 1998),'Principles and Applications of GSM', Prentice Hall PTR

[8] Ziad A. Osman, Mazen Jrab, Souleiman Midani, Rached N. Zantout (May 2003),

[9]_Implementation of a System for Offline Tracking using GPS`,Mediterranean Microwave

[10] Symposium, Ain Shams University, Cairo,Egypt, pp. 203-207. 